\title{
Clinical application of plasma mitochondrial DNA content in patients with lung cancer
}

\author{
JIANHUA CHEN ${ }^{1}$, LEMENG ZHANG ${ }^{1}$, XUN YU $^{1}$, HUI ZHOU ${ }^{2}$, \\ YONGZHONG LUO ${ }^{1}$, WEI WANG ${ }^{1}$ and LIJING WANG ${ }^{3}$
}

\author{
${ }^{1}$ Thoracic Medicine Department 1 and ${ }^{2}$ Hematology Department, Hunan Cancer Hospital Affiliated to \\ Xiangya Medical School, Central South University, Changsha, Hunan $410013 ;{ }^{3}$ Department of Geriatrics, \\ Respiratory Medicine, Xiangya Hospital, Central South University, Changsha, Hunan 410008, P.R. China
}

Received January 14, 2018; Accepted August 29, 2018

\section{DOI: $10.3892 / \mathrm{ol} .2018 .9515$}

\begin{abstract}
Alterations of mitochondrial DNA (mtDNA) have been identified in several types of solid tumor. However, to the best of our knowledge, the clinical significance of plasma mtDNA content in lung cancer remains unknown. Thus, the current study explored the diagnostic and prognostic value of plasma mtDNA quantification in patients with lung cancer. Plasma mtDNA copy numbers of patients with lung cancer $(\mathrm{n}=128)$ and healthy individuals $(\mathrm{n}=107)$ were quantified by quantitative polymerase chain reaction. Plasma mtDNA copy numbers in patients and healthy controls were $0.89 \times 10^{4}$ and $1.37 \times 10^{4}$ copies $/ \mu 1$, respectively $(\mathrm{P}<0.0001)$. Furthermore, lower plasma mtDNA content was associated with tumor size, lymph node metastases, distant metastases and serum carcinoembryonic antigen levels $(\mathrm{P}<0.05)$, but was not associated with pathological type, age, sex or main driver gene mutation status $(\mathrm{P}>0.05)$. Plasma mtDNA facilitated the detection of lung cancer at a threshold of $1.19 \times 10^{4}$ copies $/ \mu 1$ with a sensitivity of $71.1 \%$ and specificity of $70.1 \%$, as determined by receiver operating characteristic curve analysis. Advanced stage (III and IV) patients with a lower mtDNA copy number (cutoff: $1.02 \times 10^{4}$ copies $/ \mu \mathrm{l}$ ) tended to exhibit poorer prognosis $(\mathrm{P}<0.05)$. These results indicated that plasma mtDNA content is a promising and complementary candidate with tissue mtDNA for diagnosis and prognostic prediction for lung cancer.
\end{abstract}

\section{Introduction}

Mitochondria are pivotal organelles responsible for cellular energy metabolism, free radical formation, calcium

Correspondence to: Dr Lemeng Zhang, Thoracic Medicine Department 1, Hunan Cancer Hospital Affiliated to Xiangya Medical School, Central South University, 283 Tongzipo Road, Changsha, Hunan 410013, P.R. China

E-mail: 497730787@qq.com

Key words: lung cancer, mitochondrial DNA, quantitative polymerase chain reaction, prognosis, diagnosis homeostasis and the intrinsic apoptotic pathway (1). Each mitochondrion has its own extra-chromosomal genome, termed mitochondrial DNA (mtDNA). The number of mtDNA copies is tightly controlled and remains relatively stable, which is important for maintaining homeostasis (2). However, mutations and alterations of mtDNA can lead to abnormalities in energy metabolism and oxidative stress, which may be involved in carcinogenesis (3-5).

Alterations of mtDNA content have been reported in human malignancies, but with contradictory findings. Increased mtDNA copy numbers have been identified in endometrial adenocarcinoma cells (6) and esophageal squamous cell carcinoma (7). By contrast, decreased mtDNA copy numbers have been identified in lung cancer $(8,9)$, colorectal cancer $(10)$, breast cancer (11) and renal cell carcinoma (12). Increased circulating mtDNA could be a compensatory response to decreased respiratory function (13) and may be associated with increased tumor burden, increased mitochondrial damage, or release from apoptotic and/or necrotic cancer cells in cancerous lesions $(14,15)$. Conversely, decreased mtDNA content may be a consequence of exposure to excessive reactive oxygen species (16).

Compared with nuclear DNA, the quantification of mtDNA has several advantages, including short length, simple molecular structure and abundance. However, assessing mtDNA in tumor tissues requires invasive techniques. For dynamic patient management, a non-invasive diagnostic and prognostic biomarker is required (14). The monitoring of circulating mtDNA is non-invasive, convenient and suitable for dynamic observation, which makes it an ideal candidate for clinical application. Circulating mtDNA has been considered a biomarker in numerous types of tumor. As with tissue mtDNA, serum mtDNA values vary among malignancies and are increased in patients with urologic malignancies (17), epithelial ovarian cancer (18), gastric cancer (19) and testicular cancer (20), but are decreased in patients with breast cancer (21) and Ewing's sarcoma (22).

The high morbidity and mortality of lung cancer (23) has prompted interest in the study of circulating mtDNA. Hou et al (24) have reported that serum mtDNA is increased in patients with lung cancer. However, associations between mtDNA content and tumor size, lymph node metastases, distant metastases, driver gene mutation status, chemo-sensitivity 
and prognosis are still largely unknown. Given the numerous potential interactions between mtDNA and carcinogenesis, the current study hypothesized that alterations of plasma mtDNA content may be a biomarker for the presence and development of lung cancer. Thus, using quantitative polymerase chain reaction (qPCR) assays, the current study measured the copy number of plasma mtDNA in patients with lung cancer. Understanding the associations between plasma mtDNA content and the clinicopathological characteristics and prognosis of lung cancer may allow for a non-invasive and dynamic means of evaluating the disease.

\section{Materials and methods}

Patients, study design and sample collection. A total of 128 patients with lung cancer and 107 well-matched healthy controls from Medical Examination Center were included in the current study. In the lung cancer group, there are 72 males and 56 female patients with a median age of $55.2 \pm 7.8$ years. In the healthy control group, there are 57 male and 50 female individuals with a median age of $53.5 \pm 8.8$ years. The patients with lung cancer were diagnosed from January to December 2015 at the Hunan Cancer Hospital of China (Changsha, China). The present study was approved by the Ethics Committee of Hunan Cancer Hospital. Informed consent was obtained from healthy controls and each patient according to protocols approved by the hospital's Ethics Committees. None of the patients had received pre-operative radiotherapy or chemotherapy. The exclusion criteria included autoimmune diseases, inherited diseases, mitochondrial-related diseases, or patients who refused to provide informed consent. Whole blood was collected in EDTA anticoagulant tubes and samples were centrifuged at $400 \mathrm{x}$ g at $4^{\circ} \mathrm{C}$ for $2 \mathrm{~h}$. The plasma samples were collected and stored at $-80^{\circ} \mathrm{C}$ until use. A case-control study was conducted to evaluate the associations between mtDNA copy number and clinical characteristics, and to retrospectively explore the diagnostic and prognostic value of mtDNA content. The central laboratory of Hunan Cancer Hospital measured serum tumor biomarkers. The TNM classification was according to National Comprehensive Cancer Network Classification Standard; Seventh Edition (25). The following categories of patients were enrolled for analysis of progression-free survival (PFS): Advanced lung cancer (stage III and IV); Eastern Cooperative Oncology Group performance-status score of 0 or 1 (on a 5-point scale) (26); and measurable disease according to the Response Evaluation Criteria in Solid Tumors, version 1.1 (27). Enrolled patients had received no primary systemic chemotherapy for advanced or metastatic disease. Epidermal growth factor receptor (EGFR) mutation was detected by direct sequencing and anaplastic lymphoma kinase (ALK) mutation was detected by immunohistochemistry as described previously (28). Patients with driver gene mutations who had received EGFR-TKI or crizotinib were excluded from analysis of PFS. PFS was defined as the length of time during and following primary treatment of lung cancer when a patient lived with the disease but without progression, as demonstrated by radiological and clinical examinations.

DNA isolation and $q P C R$. Total plasma DNA was isolated with a QIAamp DNA Blood Mini kit (Qiagen, Inc., Valencia,
CA, USA) according to the manufacturer's protocol. The mtDNA content was measured using qPCR as described previously (29). The mtDNA plasmid was constructed by YRBIO (Changsha, China) and a standard curve was generated using six dilutions of DNA $\left(10^{1}-10^{6}\right.$ copies $\left./ \mu \mathrm{l}\right)$. The ratio of mitochondrial ND1 to human 36B4 was used. Forward ND1 primer: 5'-CCCTAAAACCCGCCACATCT-3'; reverse ND1 primer: 5'-GAGCGATGGTGAGAGCTAAGGT-3'; forward human 36B4 primer: 5'-CAGCAAGTGGGAAGGTGTAATCC-3'; reverse human 36B4 primer: 5'-CCCATTCTATCATCAACG GGTACAA-3'. qPCR was applied to $20-\mu 1$ reaction volumes containing $2 \mu \mathrm{l}$ DNA, $10 \mu \mathrm{l}$ PCR Master Mix (Toyobo Life Science, Osaka, Japan), $4 \mu 1$ primers, $3.6 \mu 1$ double-distilled water and $0.4 \mu \mathrm{l}$ ROX dye (Qiagen, Inc., Valencia, CA, USA). PCR was performed as follows: $95^{\circ} \mathrm{C}$ for $60 \mathrm{sec}$, followed by $95^{\circ} \mathrm{C}$ for $15 \mathrm{sec}$ and $60^{\circ} \mathrm{C}$ for $45 \mathrm{sec}$, repeated for 40 cycles. Relative gene expression was calculated using the comparative $2^{=\Delta \Delta \mathrm{Cq}}$ method (30).

Statistical analysis. The SPSS statistical package was used for all analyses (version 22.0, IBM Corp., Armonk, NY, USA). Data are presented as the mean \pm standard deviation. The Kolomogorov-Smirnov test was used to check whether the samples independent exhibited a normal distribution. For normal distributions, a paired Student's t-test was used for two groups, and one-way analysis of variance followed by Tukey's post-hoc test was used for comparison of multiple groups. For variables not in a normal distribution, unpaired samples were compared by use of the Mann-Whitney U test, and multiple independent samples were compared with the Kruskall-Wallis $\mathrm{H}$ test with Tukey's post-hoc test. A receiver operating characteristic (ROC) curve was used to analyze the diagnostic applicability of plasma mtDNA with the Youden index for identification of the optimal cut-off point. The Kaplan-Meier method was used for prognostic analysis. The log-rank test was used. Spearman's correlation coefficient was used to calculate the correlation between carcinoembryonic antigen (CEA) and mtDNA copy number. It also was used to analyze the correlation with age. $\mathrm{P}<0.05$ was considered to indicate a statistically significant difference.

\section{Results}

Characteristics of the study population. The characteristics of the study population are listed in Table I. A total of 128 patients with lung cancer and 107 healthy controls were enrolled, and they were well matched in terms of sex, age, and smoking history. The distributions of tumor size, lymph node metastases and distant metastases are listed. Of the cancer cases, 32 were TNM stage I, 20 were stage II, 34 were stage III and 42 were stage IV. Of the cancer cases, 54 were adenocarcinoma, 44 were squamous cell carcinoma and 30 were small-cell lung cancer. A total of 29 patients had epidermal growth factor receptor (EGFR) mutations and 9 had an anaplastic lymphoma kinase (ALK) fusion.

Distribution of plasma mtDNA content in patients with lung cancer and healthy controls. A qPCR assay was performed to explore the distribution of plasma mtDNA content in patients with lung cancer and healthy control subjects. As illustrated 
Table I. Characteristics of study population.

\begin{tabular}{|c|c|c|c|}
\hline Characteristics & $\begin{array}{l}\text { Lung } \\
\text { cancer } \\
(n=128)\end{array}$ & $\begin{array}{l}\text { Healthy } \\
\text { control } \\
(n=107)\end{array}$ & P-value \\
\hline Sex, $\mathrm{n}$ & & & 0.694 \\
\hline Male & 72 & 57 & \\
\hline Female & 56 & 50 & \\
\hline Age, years $($ mean \pm SD) & $55.2 \pm 7.8$ & $53.5 \pm 8.8$ & 0.125 \\
\hline Smoking history, $\mathrm{n}$ & & & 0.703 \\
\hline Yes & 69 & 55 & \\
\hline No & 59 & 52 & \\
\hline \multicolumn{4}{|l|}{ Tumor size, $\mathrm{n}$} \\
\hline $\mathrm{T} 1$ & 32 & & \\
\hline $\mathrm{T} 2$ & 44 & & \\
\hline T3 & 22 & & \\
\hline $\mathrm{T} 4$ & 30 & & \\
\hline \multicolumn{4}{|l|}{ Lymph node metastasis, $\mathrm{n}$} \\
\hline No & 38 & & \\
\hline N1 & 22 & & \\
\hline $\mathrm{N} 2$ & 20 & & \\
\hline N3 & 48 & & \\
\hline \multicolumn{4}{|l|}{ TNM stage, $\mathrm{n}$} \\
\hline I & 32 & & \\
\hline II & 20 & & \\
\hline III & 34 & & \\
\hline IV & 42 & & \\
\hline \multicolumn{4}{|l|}{ Pathology type, $\mathrm{n}$} \\
\hline Adenocarcinoma & 54 & & \\
\hline Squamous cell carcinoma & 44 & & \\
\hline Small cell lung cancer & 30 & & \\
\hline Main driver gene mutation & & & \\
\hline EGFR & 29 & & \\
\hline ALK & 9 & & \\
\hline
\end{tabular}

EGFR, epidermal growth factor receptor; ALK, anaplastic lymphoma kinase.

in Fig. 1, plasma mtDNA content in patients with lung cancer was significantly lower compared with that in healthy controls $\left(0.89 \times 10^{4}\right.$ and $1.37 \times 10^{4}$ copies $/ \mu 1$, respectively; $\left.\mathrm{P}<0.0001\right)$.

Associations between plasma mtDNA content and clinicopathological characteristics of lung cancer. The associations between plasma mtDNA content and clinicopathological characteristics of lung cancers were also evaluated. As illustrated in Fig. 2A-E, plasma mtDNA content was negatively associated with tumor size (T3 and T4 vs. T1, P<0.05), lymph node metastases (N1-3 vs. N0, P<0.05), distant metastases (M1 vs. M0, P<0.0001) and TNM stage (III and IV vs. I, P<0.01; III/IV vs. I/II, P<00001). As illustrated in Fig. 2F, mtDNA contents were significantly higher in patients with smoking history compared with in non-smokers $(\mathrm{P}=0.0029)$. However, mtDNA contents were not associated with pathological type (Fig. 3A)

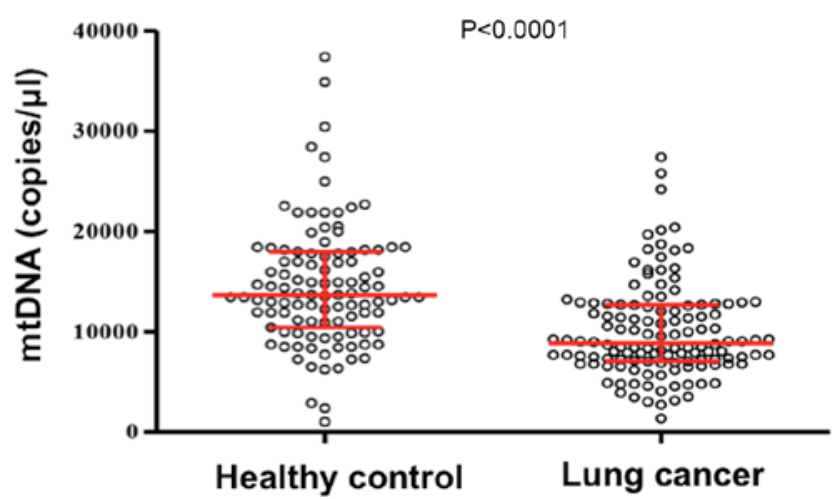

Figure 1. Distribution of plasma mtDNA content in patients with lung cancer and healthy controls. Quantitative polymerase chain reaction was used to determine plasma mtDNA copy number. Each circle represents plasma mtDNA content of each case. The horizontal lines represent the median and interquartile range and were compared with Student's t-test. mtDNA, mitochondrial DNA.

or sex (Fig. 3B), and were not correlated with age (Fig. 3C) $(\mathrm{P}>0.05)$.

Associations between plasma mtDNA content and driver gene mutation status and serum tumor biomarkers. There is a strong association between driver gene mutations and the progression of lung cancer (31). The current study analyzed plasma mtDNA content in patients with or without epidermal growth factor receptor/anaplastic lymphoma kinase (EGFR/ALK) mutations. The results revealed that plasma mtDNA content in patients with EGFR/ALK mutations was not significantly different compared with patients without EGFR/ALK mutations $(\mathrm{P}=0.0777)$. As demonstrated in Fig. 4B, CEA levels were negatively correlated with plasma mtDNA copy number $(\mathrm{r}=-0.3820, \mathrm{P}<0.0001)$. Serum tumor biomarkers, neuron-specific enolase, carbohydrate antigen-125 and carbohydrate antigen-199, were not correlated with plasma mtDNA content (data not shown).

Diagnostic value of mtDNA in patients with lung cancer. An ROC curve was used to analyze the diagnostic applicability of plasma mtDNA. As demonstrated in Fig. 5, plasma mtDNA facilitated the detection of lung cancer at a cutoff value of $1.19 \times 10^{4}$ copies $/ \mu 1$ with a sensitivity of $71.1 \%$ and specificity of $70.1 \%$. With this cutoff value, the diagnostic accuracy of plasma mtDNA is $71.1 \%$, the positive predictive value is $74.2 \%$, and the negative predictive value is $67.6 \%$. This finding suggests promise for mtDNA as a reliable test for lung cancer.

Prognostic value of mtDNA in advanced lung cancer patients. mtDNA content has previously been associated with prognosis in patients with colon cancer and gastric cancer at an advanced stage $(32,33)$. To explore the prognostic value of mtDNA in patients with lung cancer, the current study determined the PFS in patients with advanced stage (III and IV) cancer divided into two groups based on mtDNA cutoff point of $1.02 \times 10^{4}$ copies $/ \mu$ l. As illustrated in Fig. 6, PFS was longer in patients with mtDNA content higher than the cutoff point compared with patients with mtDNA content lower than the 
A

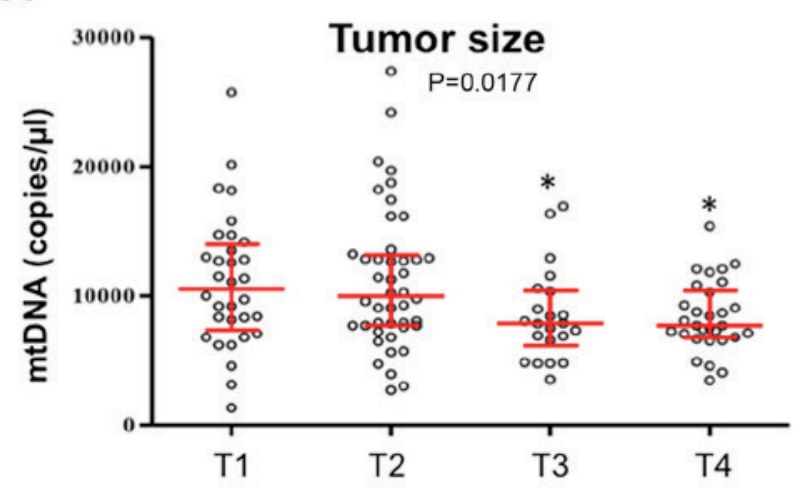

B

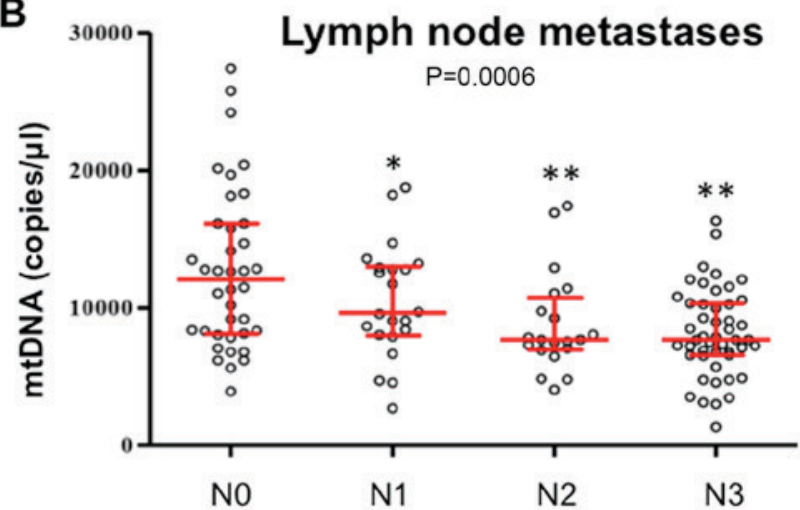

C

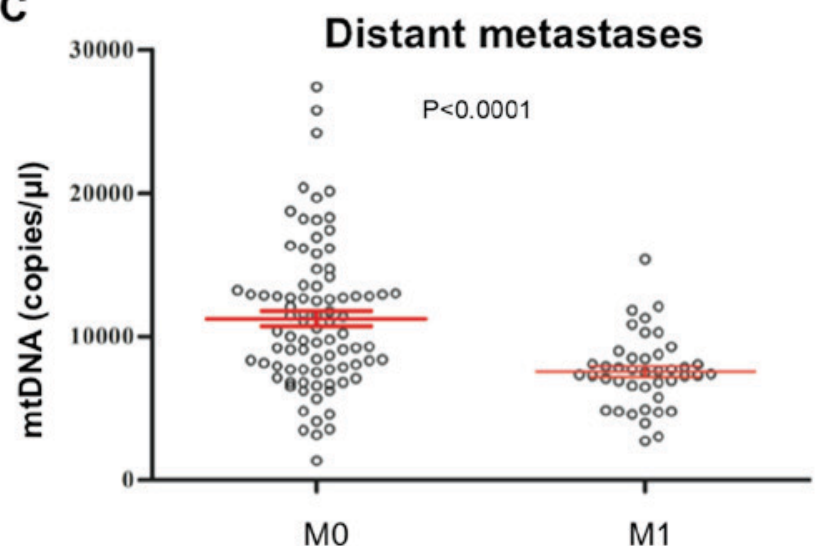

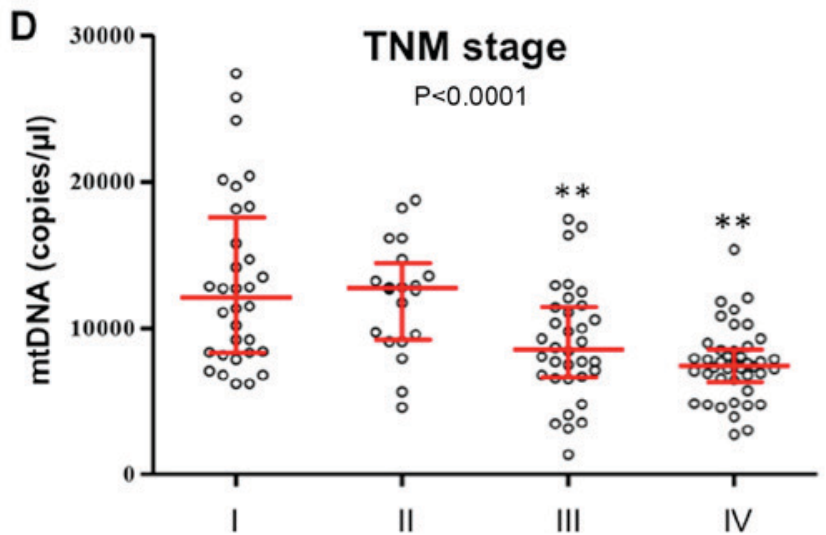

E

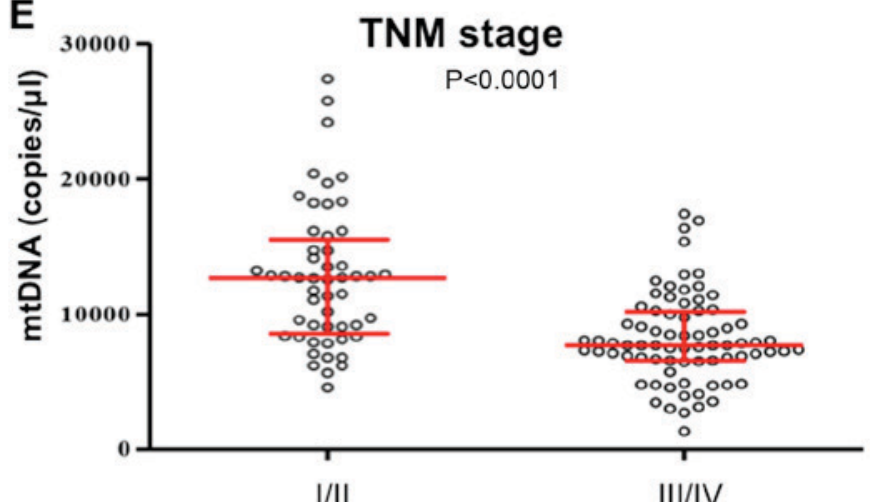

F

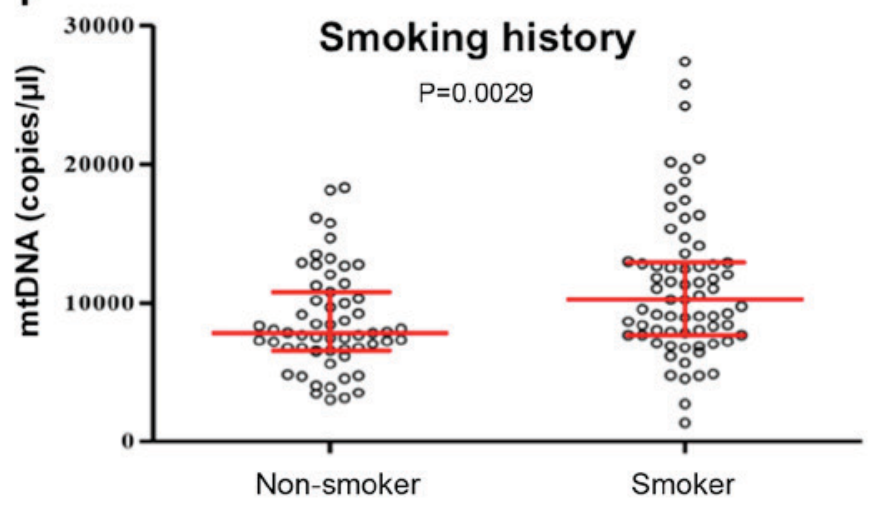

Figure 2. Associations between plasma mtDNA content and (A) tumor size, (B) lymph node metastases, (C) distant metastases, (D) TNM stage I-IV, (E) TNM stage I/II vs. III/IV, and (F) smoking history in patients with lung cancer. Each circle represents the plasma mtDNA content of each case. The horizontal lines represent the median and interquartile range. Student's t-test was used for two groups and analysis of variance followed by the Tukey's post hoc test was used for comparison of multiple groups. ${ }^{*} \mathrm{P}<0.05$ vs. T1 (A) and vs. N0 (B); ${ }^{* *} \mathrm{P}<0.01$ vs. N0 (B) and vs. stage I (D). mtDNA, mitochondrial DNA.

cutoff point, as assessed by Kaplan-Meier curve analysis (7.8 vs. 6.9 months; $\mathrm{P}<0.05)$.

\section{Discussion}

The morbidity and mortality of lung cancer are still extremely high for patients with malignant tumors. There were an estimated 222,500 new cases and 155,870 deaths in the United States in 2017 (34). Lack of methods for early diagnosis and effective treatment are at least partly responsible for the low 5-year survival rate (35). Although quantitative analyses have focused on the identification of biomarkers for lung cancer, there is still a need for novel, specific biomarkers for early detection, prognosis and dynamic observation.

Energy metabolism reprogramming is an important characteristic of cancer (36). One of the underlying mechanisms is mitochondrial dysfunction caused by disorders of structure and of the mitochondrial genome (36). Abnormalities of mtDNA may lead to increased reactive oxygen species, disrupted calcium homeostasis, reprogramed metabolism and resistance to apoptosis (37). Changes in mtDNA content have been reported in malignancies, with both down- and upregulation of mtDNA content in solid tumors. Decreased mtDNA content has been associated with renal cell carcinoma (38), hepatocellular carcinoma (39) and esophageal 

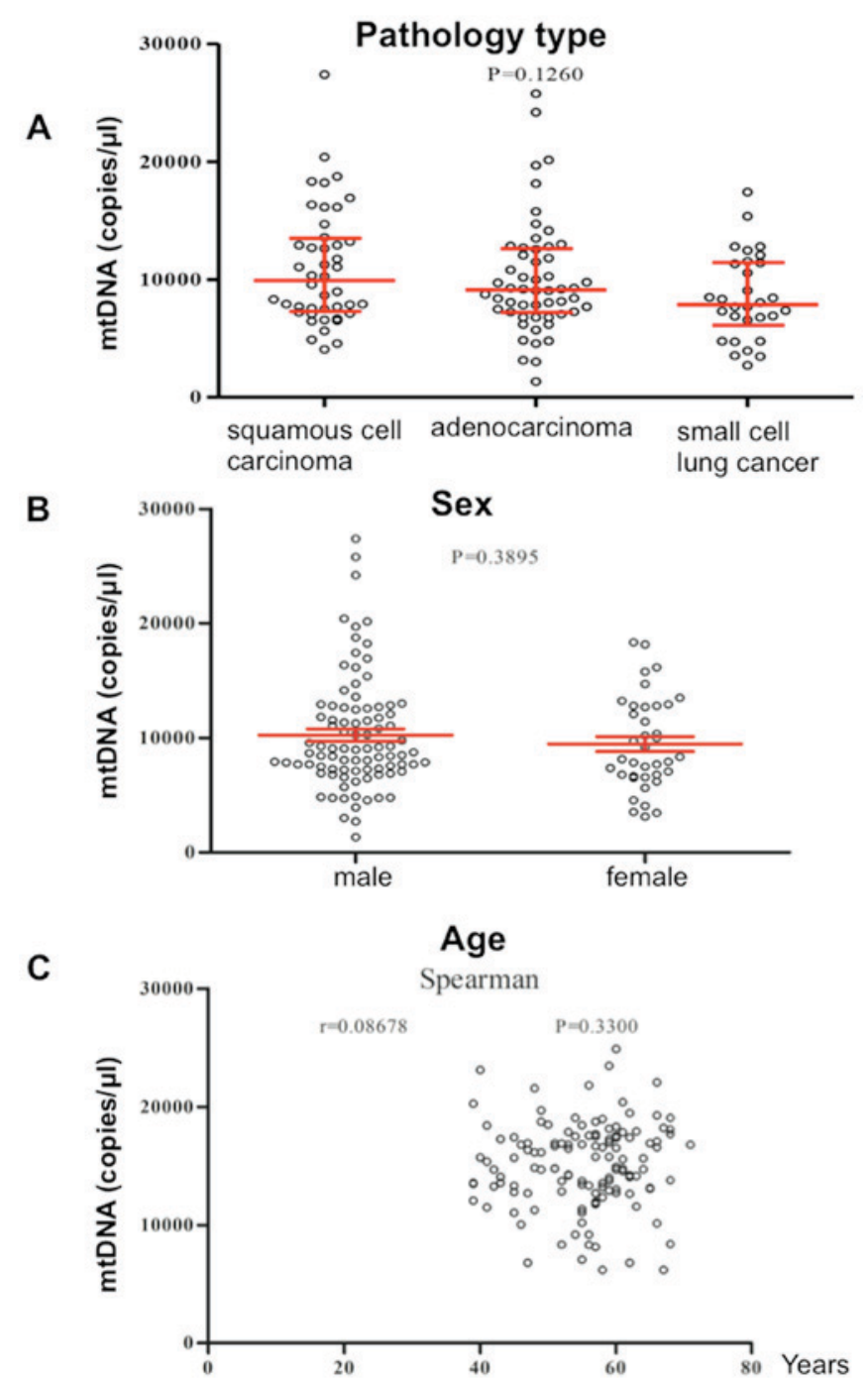

Figure 3. Associations between plasma mtDNA content and (A) pathological type, (B) sex, and age (C) in patients with lung cancer. Each circle represents the plasma mtDNA content of each case. The horizontal lines represent the median and interquartile range. Student's t-test was used for two groups and analysis of variance followed by the Tukey's post hoc test was used for comparison of multiple groups. Correlation analysis between mtDNA content and age was performed by Spearman's correlation coefficient. mtDNA, mitochondrial DNA.

adenocarcinoma (40). By contrast, increased mtDNA content in pancreatic cancer (41), colorectal cancer (42) and breast cancer (43) has been reported. Since mtDNA may be involved in carcinogenesis, oncologists and pulmonologists have been motivated to monitor its dynamic alterations in patients with lung cancer. Hou et al (24) reported increased serum mtDNA content, while others have revealed significantly reduced mtDNA content in lung cancer tissues $(8,9)$. In the current study, it was identified that circulating mtDNA content was significantly lower in patients with lung cancer compared with healthy subjects. The lower mtDNA content may reflect the reduced capacity of compensatory responses to oxidative stress damage (44). The decrease in mtDNA may also be a consequence of mutation or depletion in the mtDNA D-loop caused by reactive oxygen species (45).

Alterations in somatic mtDNA copy number have been revealed to strongly correlate with clinicopathological characteristics, early diagnosis, progression, and radiotherapy and chemotherapy efficacy in malignancies (14). The current study identified that lower plasma mtDNA contents were negatively associated with tumor size, lymph node metastases, distant metastases and serum CEA level, but were not associated with age, sex, pathological type or main driver gene mutation status. The incidence of D-loop mutations of mtDNA in advanced cancer stages has been revealed to be higher when compared with patients in the early stage of disease (8), which may lead to lower mtDNA copy numbers in patients with late-stage cancer. These observations may account for the current findings that plasma mtDNA content was negatively associated with tumor size, lymph node metastases and distant metastases.

Serum tumor biomarkers including CEA have been widely used to monitor the progression of tumors (46). In the current study, a negative correlation between plasma mtDNA content and serum CEA level was revealed, which to the best of our knowledge, is the first time this correlation has been recorded. Both serum CEA levels and plasma mtDNA can be dynamically monitored for chemotherapeutic effects and disease progression (46). Thus, the combined determination of CEA and mtDNA content may improve the efficacy and prognostic ability of these tests in lung cancer. In related work, no relationship between mtDNA content and serum prostate-specific antigen levels has been identified in prostate cancer and epithelial ovarian cancer $(18,47)$. Further studies should be performed to explore the predictive value of the combination of dynamic monitoring of CEA level and circulating mtDNA alteration during chemotherapy. However, the efficacy of chemotherapy in multiple solid tumors is affected by a number of factors (48). A perspective study, with these relevant factors well matched, is required to explore the predictive value of dynamic circulating mtDNA content.

The quantification of plasma mtDNA may be used to recognize patients with poor prognosis, similar to the lower mtDNA copy number in patients who have advanced breast cancer, glioma and prostate cancer $(32,33,49,50)$. Lower mtDNA content has been identified to be associated with tumor progression in lung cancer tissues following chemotherapy (51). Variations in mtDNA have been reported to be associated with radiation-induced toxicity (52). The change of mtDNA content can also improve the cytotoxicity of chemotherapeutic agents. DNA damage in mitochondria alters the mitochondrial apoptotic signaling pathway, thereby promoting the survival of cancer cells and even changing their resistance to anticancer drugs (53). Radiotherapy and chemotherapy are the most important treatments for advanced lung cancer. Clinically, sensitivity to radiotherapy and chemotherapy are determining factors for prognosis. Given that mtDNA depletion affects radiotherapy and chemotherapy sensitivity (5), the current study further explored the prognostic value of lower plasma mtDNA content in advanced lung cancer patients. As previously reported (54), the current study revealed that a lower mtDNA copy number was associated with poor prognosis in patients with advanced stage cancer, which suggests that plasma mtDNA copy number is a promising prognostic candidate for lung cancer.

Further studies are required to elucidate the mechanisms and consequences of decreased mtDNA content in lung cancer 

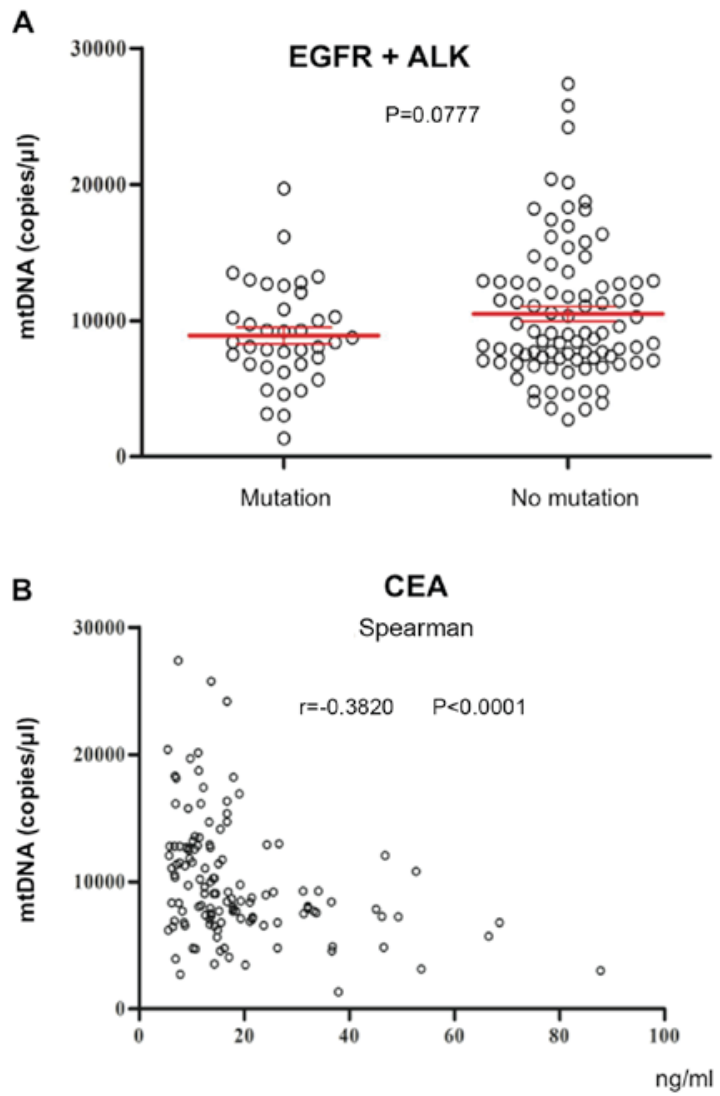

Figure 4. Association between plasma mtDNA content and (A) driver gene mutation status and (B) serum tumor biomarkers. Each circle represents the plasma mtDNA content of each case. The horizontal lines represent median and interquartile range and were determined with the Mann-Whitney U test. Spearman's correlation coefficient was used to calculate the correlation between CEA and mtDNA copy number. ALK, anaplastic lymphoma kinase; CEA, carcinoembryonic antigen; EGFR, epidermal growth factor receptor; mtDNA, mitochondrial DNA.

carcinogenesis. The lower mtDNA copy number may alter mitochondrial gene expression and lead to decreased mitochondrial function and energy metabolism and cause increased generation of reactive oxygen species $(55,56)$, enhanced glycolysis, and even alterations in mitochondrial bioenergetic and biosynthetic states (57). Decreased mtDNA may also promote epithelial-mesenchymal transition (58-60) and apoptosis-resistant cancer cells through the phosphoinositide 3-kinase/Akt signaling pathway (61). Changes in mtDNA copy number may explain the persistent deficiency in mitochondrial activity. Thus, the mtDNA-depleted $\varrho 0$ cell line may be used to further explore the consequences and mechanism of mtDNA deficiency in lung cancer carcinogenesis.

To the best of our knowledge, the current study is the largest that has explored the diagnostic, predictive and prognostic clinical application of plasma mtDNA content in patients with lung cancer. The current study is also the most comprehensive study of plasma mtDNA alteration in patients with lung cancer. However, further studies of specific subgroups with large sample sizes are still required to further confirm the subgroup conclusions made by the current study.

In conclusion, decreased plasma mtDNA content was associated with tumor metastatic potential and unfavorable

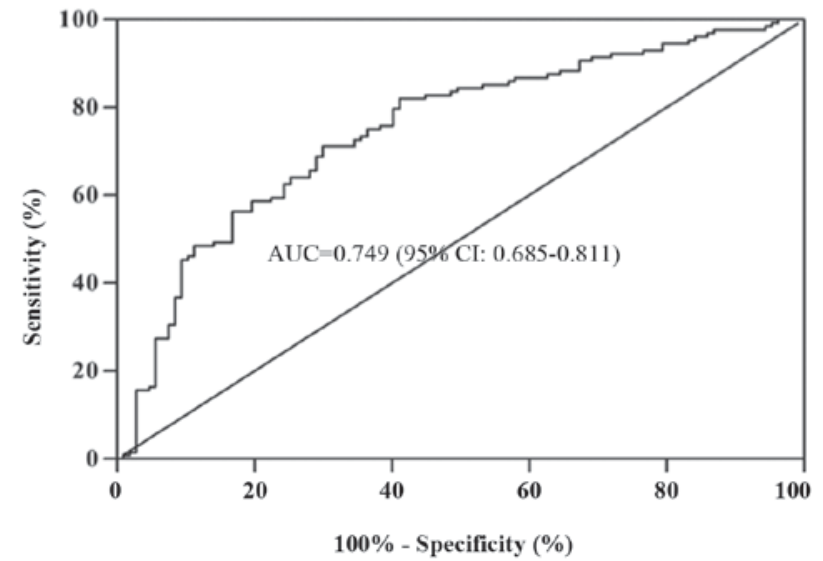

Figure 5. Receiver operating characteristic curve analysis to determine the sensitivity and specificity of mitochondrial DNA copy number in patients with lung cancer. CI, confidence interval; AUC, area under the curve.

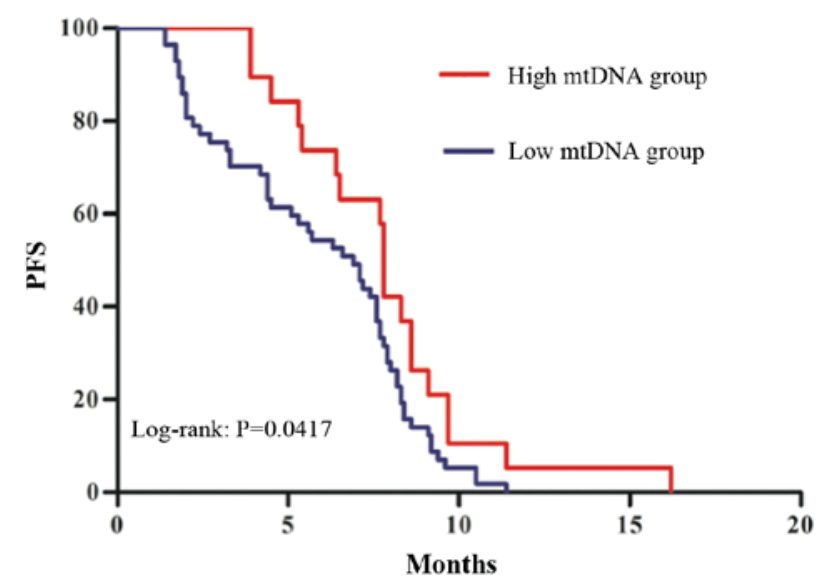

Figure 6. Kaplan-Meier survival analysis of mtDNA copy number for estimating prognosis in patients with advanced lung cancer. The median mtDNA copy number $\left(1.02 \times 10^{4}\right.$ copies $\left./ \mu 1\right)$ was set as the cutoff point. PFS, progression-free survival; mtDNA, mitochondrial DNA.

prognosis in patients with lung cancer. Monitoring circulating mtDNA content is a promising approach for diagnosis and prognosis of lung cancer. Further studies of the epigenetic alterations of mtDNA are required to understand its downstream effectors and role in lung cancer pathogenesis. Although translating plasma mtDNA quantification into routine clinical practice may take several steps, knowledge regarding the potential applicability of circulating mtDNA quantification in the diagnosis and prognosis of lung cancer has progressed considerably.

\section{Acknowledgements}

Not applicable.

\section{Funding}

The present study was supported by grant from the National Natural Science Foundation of China (grant no. 81401631) and by grant from Key Research and Develop Program of Hunan Province, China (grant no. 2017WK2061). 


\section{Availability of data and materials}

All data generated or analyzed during the present study are included in this published article.

\section{Authors' contributions}

JC and LZ conceived and designed the experiments; LZ, XY, $\mathrm{HZ}$ and $\mathrm{YL}$ performed the experiments and contributed to molecular analysis; WW and LW analyzed the data and LZ wrote the manuscript.

\section{Ethics approval and consent to participate}

The present study was approved by Department of Ethics Committee of Hunan Cancer Hospital (Changsha, China), and written informed consent was obtained from all participants.

\section{Patient consent for publication}

Informed consent for the publication or any associated images was obtained from healthy controls and patients according to protocols approved by the hospital's Ethics Committee.

\section{Competing interests}

The authors declare that they have no competing interests.

\section{References}

1. Wallace DC: Mitochondria and cancer. Nat Rev Cancer 12 685-698, 2012.

2. D'Erchia AM, Atlante A, Gadaleta G, Pavesi G, Chiara M, De Virgilio C, Manzari C, Mastropasqua F, Prazzoli GM, Picardi E, et al: Tissue-specific mtDNA abundance from exome data and its correlation with mitochondrial transcription, mass and respiratory activity. Mitochondrion 20: 13-21, 2015

3. Akhmedov AT and Marin-Garcia J: Mitochondrial DNA maintenance: An appraisal. Mol Cell Biochem 409: 283-305, 2015.

4. Hsu CC, Tseng LM and Lee HC: Role of mitochondrial dysfunction in cancer progression. Exp Biol Med (Maywood) 241: 1281-1295, 2016.

5. van Gisbergen MW, Voets AM, Starmans MH, de Coo IF, Yadak R, Hoffmann RF, Boutros PC, Smeets HJ, Dubois L and Lambin P: How do changes in the mtDNA and mitochondrial dysfunction influence cancer and cancer therapy? Challenges, opportunities and models. Mutat Res Rev Mutat Res 764: 16-30, 2015.

6. Wang Y, Liu VW, Xue WC, Tsang PC, Cheung AN and Ngan HY: The increase of mitochondrial DNA content in endometrial adenocarcinoma cells: A quantitative study using laser-captured microdissected tissues. Gynecol Oncol 98: 104-110, 2005.

7. Lin CS, Lee HT, Lee SY, Shen YA, Wang LS, Chen YJ and Wei YH: High mitochondrial DNA copy number and bioenergetic function are associated with tumor invasion of esophageal squamous cell carcinoma cell lines. Int J Mol Sci 13: 11228-11246, 2012.

8. Lee HC, Yin PH, Lin JC, Wu CC, Chen CY, Wu CW, Chi CW, Tam TN and Wei YH: Mitochondrial genome instability and mtDNA depletion in human cancers. Ann N Y Acad Sci 1042: 109-122, 2005

9. Dai JG, Zhang ZY, Liu QX and Min JX: Mitochondrial genome microsatellite instability and copy number alteration in lung carcinomas. Asian Pac J Cancer Prev 14: 2393-2399, 2013.

10. Lin PC, Lin JK, Yang SH, Wang HS, Li AF and Chang SC: Expression of beta-F1-ATPase and mitochondrial transcription factor A and the change in mitochondrial DNA content in colorectal cancer: Clinical data analysis and evidence from an in vitro study. Int J Colorectal Dis 23: 1223-1232, 2008.

11. Fan AX, Radpour R, Haghighi MM, Kohler C, Xia P, Hahn S, Holzgreve W and Zhong X: Mitochondrial DNA content in paired normal and cancerous breast tissue samples from patients with breast cancer. J Cancer Res Clin Oncol 135: 983-989, 2009.
12. Meierhofer D, Mayr JA, Foetschl U, Berger A, Fink K, Schmeller N, Hacker GW, Hauser-Kronberger C, Kofler B and Sperl W: Decrease of mitochondrial DNA content and energy metabolism in renal cell carcinoma. Carcinogenesis 25: 1005-1010, 2004.

13. Hosgood HD III, Liu CS, Rothman N, Weinstein SJ, Bonner MR, Shen M, Lim U, Virtamo J, Cheng WL, Albanes D and Lan Q: Mitochondrial DNA copy number and lung cancer risk in a prospective cohort study. Carcinogenesis 31: 847-849, 2010.

14. Yu M: Circulating cell-free mitochondrial DNA as a novel cancer biomarker: Opportunities and challenges. Mitochondrial DNA 23: 329-332, 2012

15. Strelkova I, Abdullaev SA, Snigireva GP, Bezlepkin VG and Gaziev AI: Share of extracellular mutated mitochondrial DNA increases in plasma of lung cancer patients following radiotherapy. Biomed Khim 56: 517-525, 2010.

16. Shimura $T$ and Kunugita N: Mitochondrial reactive oxygen species-mediated genomic instability in low-dose irradiated human cells through nuclear retention of cyclin D1. Cell Cycle 15: 1410-1414, 2016.

17. Ellinger J, Muller DC, Muller SC, Hauser S, Heukamp LC, von Ruecker A, Bastian PJ and Walgenbach-Brunagel G: Circulating mitochondrial DNA in serum: A universal diagnostic biomarker for patients with urological malignancies. Urol Oncol 30: 509-515, 2012.

18. Zachariah RR, Schmid S, Buerki N, Radpour R, Holzgreve W and Zhong X: Levels of circulating cell-free nuclear and mitochondrial DNA in benign and malignant ovarian tumors. Obstet Gynecol 112: 843-850, 2008.

19. Fernandes J, Michel V, Camorlinga-Ponce M, Gomez A, Maldonado C, De Reuse H, Torres J and Touati E: Circulating mitochondrial DNA level, a noninvasive biomarker for the early detection of gastric cancer. Cancer Epidemiol Biomarkers Prev 23: 2430-2438, 2014.

20. Ellinger J, Albers P, Muller SC, von Ruecker A and Bastian PJ: Circulating mitochondrial DNA in the serum of patients with testicular germ cell cancer as a novel noninvasive diagnostic biomarker. BJU Int 104: 48-52, 2009.

21. Kohler C, Radpour R, Barekati Z, Asadollahi R, Bitzer J, Wight E, Bürki N, Diesch C, Holzgreve W and Zhong XY: Levels of plasma circulating cell free nuclear and mitochondrial DNA as potential biomarkers for breast tumors. Mol Cancer 8: 105, 2009.

22. Yu M, Wan YF and Zou QH: Cell-free circulating mitochondrial DNA in the serum: A potential non-invasive biomarker for Ewing's sarcoma. Arch Med Res 43: 389-394, 2012.

23. Siegel RL, Miller KD and Jemal A: Cancer statistics, 2016. CA Cancer J Clin 66: 7-30, 2016.

24. Hou YL, Chen JJ, Wu YF, Xue CJ, Li FZ, Zheng Q and Chen H: Clinical significance of serum mitochondrial DNA in lung cancer. Clin Biochem 46: 1474-1477, 2013.

25. Rusch VW, Asamura H, Watanabe H, Giroux DJ, Rami-Porta R, Goldstraw P and Members of IASLC Staging Committee: The IASLC lung cancer staging project: A proposal for a new international lymph node map in the forthcoming seventh edition of the TNM classification for lung cancer. J Thorac Oncol 4: 568-577, 2009.

26. Prigerson HG, Bao Y, Shah MA, Paulk ME, LeBlanc TW, Schneider BJ, Garrido MM, Reid MC, Berlin DA, Adelson KB, et al: Chemotherapy use, performance status, and quality of life at the end of life. JAMA Oncol 1: 778-784, 2015.

27. Eisenhauer EA, Therasse P, Bogaerts J, Schwartz LH, Sargent D, Ford R, Dancey J, Arbuck S, Gwyther S, Mooney M, et al: New response evaluation criteria in solid tumours: Revised RECIST guideline (version 1.1). Eur J Cancer 45: 228-247, 2009.

28. Garassino MC, Cho BC, Kim JH, Mazières J, Vansteenkiste J, Lena H, Corral Jaime J, Gray JE, Powderly J, Chouaid C, et al: Durvalumab as third-line or later treatment for advanced non-small-cell lung cancer (ATLANTIC): An open-label, single-arm, phase 2 study. Lancet Oncol 19: 521-536, 2018.

29. Li L, Hann HW, Wan S, Hann RS, Wang C, Lai Y, Ye X, Evans A, Myers RE, Ye Z, et al: Cell-free circulating mitochondrial DNA content and risk of hepatocellular carcinoma in patients with chronic HBV infection. Sci Rep 6: 23992, 2016.

30. Livak KJ and Schmittgen TD: Analysis of relative gene expression data using real-time quantitative PCR and the $2-\Delta \Delta \mathrm{Ct}$ method. Methods 25: 402-408, 2001.

31. Campbell JD, Alexandrov A, Kim J, Wala J, Berger AH, Pedamallu CS, Shukla SA, Guo G, Brooks AN, Murray BA, et al: Distinct patterns of somatic genome alterations in lung adenocarcinomas and squamous cell carcinomas. Nat Genet 48: 607-616, 2016. 
32. Wang Y, He S, Zhu X, Qiao W and Zhang J: High copy number of mitochondrial DNA predicts poor prognosis in patients with advanced stage colon cancer. Int J Biol Markers 31: e382-e388, 2016.

33. Zhang G, Qu Y, Dang S, Yang Q, Shi B and Hou P: Variable copy number of mitochondrial DNA (mtDNA) predicts worse prognosis in advanced gastric cancer patients. Diagn Pathol 8: 173, 2013.

34. Siegel RL, Miller KD and Jemal A: Cancer Statistics, 2017. CA Cancer J Clin 67: 7-30, 2017

35. Chen W, Zheng R, Baade PD, Zhang S, Zeng H, Bray F, Jemal A, Yu XQ and He J: Cancer statistics in China, 2015. CA Cancer J Clin 66: 115-132, 2016

36. Hanahan D and Weinberg RA: Hallmarks of cancer: The next generation. Cell 144: 646-674, 2011.

37. Sabharwal SS and Schumacker PT: Mitochondrial ROS in cancer: Initiators, amplifiers or an Achilles' heel? Nat Rev Cancer 14: 709-721, 2014.

38. Hofmann JN, Hosgood HD III, Liu CS, Chow WH, Shuch B, Cheng WL, Lin TT, Moore LE, Lan Q, Rothman N and Purdue MP: A nested case-control study of leukocyte mitochondrial DNA copy number and renal cell carcinoma in the prostate, lung, colorectal and ovarian cancer screening trial. Carcinogenesis 35: 1028-1031, 2014.

39. Zhao S, Yang Y, Liu J, Liu H, Ge N, Yang H, Zhang H and Xing J: Association of mitochondrial DNA content in peripheral blood leukocyte with hepatitis B virus-related hepatocellular carcinoma in a Chinese Han population. Cancer science 102: 1553-1558, 2011.

40. Xu E, Sun W, Gu J, Chow WH, Ajani JA and Wu X: Association of mitochondrial DNA copy number in peripheral blood leukocytes with risk of esophageal adenocarcinoma. Carcinogenesis 34 2521-2524, 2013

41. Lynch SM, Weinstein SJ, Virtamo J, Lan Q, Liu CS, Cheng WL, Rothman N, Albanes D and Stolzenberg-Solomon RZ: Mitochondrial DNA copy number and pancreatic cancer in the alpha-tocopherol beta-carotene cancer prevention study. Cancer Prev Res (Phila) 4: 1912-1919, 2011

42. Qu F, Liu X, Zhou F, Yang H, Bao G, He X and Xing J: Association between mitochondrial DNA content in leukocytes and colorectal cancer risk: A case-control analysis. Cancer 117: 3148-3155, 2011.

43. Shen J, Platek M, Mahasneh A, Ambrosone CB and Zhao $\mathrm{H}$ Mitochondrial copy number and risk of breast cancer: A pilot study. Mitochondrion 10: 62-68, 2010.

44. von Kleist-Retzow JC, Hornig-Do HT, Schauen M, Eckertz S Dinh TA, Stassen F, Lottmann N, Bust M, Galunska B, Wielckens $\mathrm{K}$, et al: Impaired mitochondrial $\mathrm{Ca} 2+$ homeostasis in respiratory chain-deficient cells but efficient compensation of energetic disadvantage by enhanced anaerobic glycolysis due to low ATP steady state levels. Exp Cell Res 313: 3076-3089, 2007.

45. Lee HC, Hsu LS, Yin PH, Lee LM and Chi CW: Heteroplasmic mutation of mitochondrial DNA D-loop and 4977-bp deletion in human cancer cells during mitochondrial DNA depletion. Mitochondrion 7: 157-163, 2007.

46. Grunnet M and Sorensen JB: Carcinoembryonic antigen (CEA) as tumor marker in lung cancer. Lung Cancer 76: 138-143, 2012.

47. Zhou W, Zhu M, Gui M, Huang L, Long Z, Wang L, Chen H, Yin Y, Jiang X, Dai Y, et al: Peripheral blood mitochondrial DNA copy number is associated with prostate cancer risk and tumor burden. PLoS One 9: e109470, 2014.

48. Lissoni P, Brivio F, Fumagalli L, Messina G, Ghezzi V, Frontini L, Giani L, Vaghi M, Ardizzoia A and Gardani GS: Efficacy of cancer chemotherapy in relation to the pretreatment number of lymphocytes in patients with metastatic solid tumors. Int J Biol Markers 19: 135-140, 2004.
49. Weerts MJ, Sieuwerts AM, Smid M, Look MP, Foekens JA, Sleijfer S and Martens JW: Mitochondrial DNA content in breast cancer: Impact on in vitro and in vivo phenotype and patient prognosis. Oncotarget 7: 29166-29176, 2016.

50. Tu H, Gu J, Meng QH, Kim J, Davis JW, He Y, Wagar EA, Thompson TC, Logothetis CJ and Wu X: Mitochondrial DNA copy number in peripheral blood leukocytes and the aggressiveness of localized prostate cancer. Oncotarget 6: 41988-41996, 2015.

51. Lin CS, Wang LS, Tsai CM and Wei YH: Low copy number and low oxidative damage of mitochondrial DNA are associated with tumor progression in lung cancer tissues after neoadjuvant chemotherapy. Interact Cardiovasc Thorac Surg 7: 954-958, 2008.

52. Fachal L, Mosquera-Miguel A, Gomez-Caamano A, Sánchez-García M, Calvo P, Lobato-Busto R, Salas A and Vega A: Evaluating the role of mitochondrial DNA variation to the genetic predisposition to radiation-induced toxicity. Radiother Oncol 111: 199-205, 2014

53. Yeung M, Hurren R, Nemr C, Wang X, Hershenfeld S, Gronda M, Liyanage S, Wu Y, Augustine J,Lee EA, et al: MitochondrialDNA damage by bleomycin induces AML cell death. Apoptosis 20: 811-820, 2015.

54. Dang S, Qu Y, Wei J, Shao Y, Yang Q, Ji M, Shi B and Hou P: Low copy number of mitochondrial DNA (mtDNA) predicts worse prognosis in early-stage laryngeal cancer patients. Diagn Pathol 9: 28, 2014.

55. Cook CC, Kim A, Terao S, Gotoh A and Higuchi M: Consumption of oxygen: A mitochondrial-generated progression signal of advanced cancer. Cell death Dis 3: e258, 2012.

56. Vermulst M, Bielas JH and Loeb LA: Quantification of random mutations in the mitochondrial genome. Methods 46: 263-268, 2008.

57. Lin CS, Lee HT, Lee MH, Pan SC, Ke CY, Chiu AW and Wei YH: Role of mitochondrial DNA copy number alteration in human renal cell carcinoma. Int J Mol Sci 17: 2016.

58. Guha M, Srinivasan S, Ruthel G, Kashina AK, Carstens RP, Mendoza A, Khanna C, Van Winkle T and Avadhani NG: Mitochondrial retrograde signaling induces epithelial-mesenchymal transition and generates breast cancer stem cells Oncogene 33: 5238-5250, 2014.

59. Naito A, Cook CC, Mizumachi T, Wang M, Xie $\mathrm{CH}$, Evans TT, Kelly T and Higuchi M: Progressive tumor features accompany epithelial-mesenchymal transition induced in mitochondrial DNA-depleted cells. Cancer Sci 99: 1584-1588, 2008.

60. Adib-Conquy $\mathbf{M}$ and Cavaillon JM: Compensatory anti-inflammatory response syndrome. Thromb Haemost 101: 36-47, 2009.

61. Moro L, Arbini AA, Yao JL, di Sant'Agnese PA, Marra E and Greco M: Mitochondrial DNA depletion in prostate epithelial cells promotes anoikis resistance and invasion through activation of PI3K/Akt2. Cell Death Differ 16: 571-583, 2009.

c) (i) $\ominus$ This work is licensed under a Creative Commons Attribution-NonCommercial-NoDerivatives 4.0 International (CC BY-NC-ND 4.0) License. 